3. Galvez-Buccollini JA, Fiestas F. Necesidad de evaluar las guías clínicas peruanas de tratamiento para trastornos mentales [carta]. Rev Peru Med Exp Salud Publica. 201 1; 28(4):698-9.

4. Gilbody S, Richards D, Brealey S, Hewitt C. Screening for depression in medical setting with the Patient Health Questionnaire (PHQ): a diagnostic meta-analysis. J Gen Intern Med. 2007;22(11):1596-602.

5. Baader T, Molina JL, Venezian S, Rojas C, Farías R, FierroFreixenet $\mathrm{C}$, et al. Validación y utilidad de la encuesta PHQ-9 (Patient Health Questionnaire) en el diagnostic de depression en pacientes usuarios de atención primaria en Chile. Rev Chil Neuro-Psiquiat. 2012;50(1):10-22.

Correspondencia: Fabian Fiestas

Dirección: Capac Yupanqui 1400, Lima 11, Perú.

Teléfono: (+511) 985-765-743

Correoelectrónico:ffiestas@epi.msu.edu

\section{NECESIDAD DE VIGILAR LA RESISTENCIA DEL $P$. falciparum AL ARTESUNATO EN EL PERÚ}

\author{
A NEED TO MONITOR P. falciparum \\ RESISTANCE TO ARTESUNATE IN PERU
}

\section{Salomón Durand ${ }^{1,2, a}$, Moisés Sihuinchaa,a, Arnaldo Lachira $^{3, a}$, Jorge Chaves ${ }^{3, a}$, César Cabezas ${ }^{4, a}$}

Sr. Editor. A finales del 2001, el Perú se convirtió en el primer país de las Américas en adoptar la terapia combinada (TC) con mefloquina/artesunato (MQ/AS) y sulfadoxina pirimetamina/artesunato (SP/AS) como terapia de primera línea para la malaria no complicada por $P$. falciparum. Posteriormente, Bolivia, Brasil, Guyana y Ecuador cambian también su esquema de tratamiento a la TC.

Ante la amenaza de la propagación de cepas resistentes a partir del sudeste asiático, en donde últimamente se ha demostrado retardo en el aclaramiento de la parasitemia y resistencia in vitro e in vivo a la artemisinina ${ }^{(1)}$, realizamos una revisión a partir de la

\footnotetext{
1 Centro de Investigación de Enfermedades Tropicales de la Marina de los EE. UU. (NAMRU-6). Iquitos, Perú.

2 Hospital de Iquitos "César Garayar García", Dirección Regional de Salud Loreto. Iquitos, Perú.

3 Dirección Regional de Salud Piura. Piura, Perú.

4 Instituto Nacional de Salud. Lima, Perú.

a Médico infectólogo-tropicalista

Recibido: 05-10-12 Aprobado: 31-10-12
}

Citar como: Durand S, Sihuincha M, Lachira A, Chaves J, Cabezas C. Necesidad de vigilar la resistencia del P. Falciparum al artesunato en el Perú. Rev Peru Med Exp Salud Publica. 2012;29(4):579-80. base datos MEDLINE, en busca de estudios realizados en nuestro país. Se buscó conocer la rapidez de acción del artesunato sobre la carga parasitaria, establecer un registro basal que sirva como punto de comparación para estudios futuros de eficacia, e identificar los factores clave donde se podría intervenir para evitar la propagación de la resistencia, en caso que esta cepa se introdujera en el Perú o para evitar que se genere en nuestra región.

Se encontraron cinco estudios sobre la eficacia de la TC en el Perú publicados entre el 2000 y 2006. El primer problema para compararlos fueron los días de seguimiento, ya que hasta el 2004, usualmente, se realizaba seguimiento por 28 días, pero en vista del prolongado tiempo de vida media de la mefloquina, posteriormente, se recomendó seguir al paciente por más de 28 días. Hasta el 2006, fecha del último estudio revisado, la TC fue muy eficaz (98-100\%), aun en el seguimiento a 63 días, y la tasa de parasitemia persistente al día 3 fue menor al 3\%. (Tabla 1).

En el estudio de Macedo et al. (2) se evaluó el esquema actual utilizado en el Perú, el cual consiste en administrar AS el primer día y MQ/AS el segundo y tercer día. Se evidenció así el efecto a las 24 horas del AS usado como monoterapia. En los otros estudios, los pacientes recibieron el esquema MQ/AS el primer y segundo día y AS el tercer día. A las 24 horas, el porcentaje de láminas positivas fue $31 \%$, y a las 48 horas (ya agregada la $M Q$ ) fue de $5 \%$. Lo hallado difiere de lo descrito por Rogers

Tabla 1. Resultados de los estudios de eficacia de TC para el tratamiento de la malaria por $P$. falciparum no complicada realizados en el Perú, 2000-2006.

\begin{tabular}{|c|c|c|c|c|c|c|c|}
\hline & \multirow[b]{2}{*}{ esquema } & \multicolumn{6}{|c|}{$\begin{array}{l}\text { Porcentaje de láminas positivas } \\
\text { durante el seguimiento }\end{array}$} \\
\hline & & $\begin{array}{c}\text { día } \\
1 \\
\end{array}$ & $\begin{array}{c}\text { día } \\
2 \\
\end{array}$ & $\begin{array}{c}\text { día } \\
3 \\
\end{array}$ & $\begin{array}{l}\text { día } \\
28 \\
\end{array}$ & $\begin{array}{l}\text { día } \\
56\end{array}$ & $\begin{array}{l}\text { día } \\
63 \\
\end{array}$ \\
\hline $\begin{array}{l}\text { Macedo et } \\
\text { al. }(2006)^{(2)}\end{array}$ & MQ/AS & $\begin{array}{l}31 / 100 \\
(31,0 \%)\end{array}$ & $\begin{array}{l}5 / 100 \\
(5,0 \%)\end{array}$ & $\begin{array}{l}1 / 100 \\
(1,0 \%)\end{array}$ & $\begin{array}{c}1 / 96 \\
(0,9 \%)^{*}\end{array}$ & - & - \\
\hline $\begin{array}{l}\text { Gutman et } \\
\text { al. }(2004)^{(3)}\end{array}$ & MQ/AS & $\begin{array}{c}3 / 38 \\
(7,8 \%)\end{array}$ & $\begin{array}{c}2 / 38 \\
(5,2 \%)\end{array}$ & $\begin{array}{c}1 / 38 \\
(2,6 \%)\end{array}$ & $0 / 38$ & $0 / 38$ & - \\
\hline \multirow[t]{2}{*}{$\begin{array}{l}\text { Grande et } \\
\text { al. }(2005)^{(4)}\end{array}$} & MQ/AS & $\begin{array}{l}108 / 260 \\
(42,5 \%)\end{array}$ & $\begin{array}{l}10 / 260 \\
(3,8 \%)\end{array}$ & 0 & - & - & $\begin{array}{l}1 / 236 \\
(0,4 \%)\end{array}$ \\
\hline & DHA/PPQ & $\begin{array}{l}84 / 260 \\
(32,3 \%)\end{array}$ & $\begin{array}{l}3 / 260 \\
(1,9 \%)\end{array}$ & 0 & - & - & $\begin{array}{l}4 / 230 \\
(1,7 \%)\end{array}$ \\
\hline $\begin{array}{l}\text { Marquino et } \\
\text { al. }(2000)^{(5)}\end{array}$ & SPIAS & - & - & $\begin{array}{c}2 / 94 \\
(2,1 \%)\end{array}$ & $\begin{array}{c}1 / 94 \\
(1,0 \%)\end{array}$ & - & - \\
\hline \multirow[t]{2}{*}{$\begin{array}{l}\text { Marquino et } \\
\text { al. }(2000)^{(6)}\end{array}$} & MQ/AS & - & $\begin{array}{c}9 / 51 \\
(17,6 \%)\end{array}$ & $\begin{array}{c}1 / 51 \\
(1,9 \%)\end{array}$ & $0 / 51$ & - & - \\
\hline & $\mathrm{MQ}$ & - & $\begin{array}{c}34 / 47 \\
(72,3 \%)\end{array}$ & $\begin{array}{c}12 / 47 \\
(25,5 \%)\end{array}$ & $0 / 47$ & - & - \\
\hline
\end{tabular}

* En el examen de genotipificación se halló que se trataba de una cepa diferente, probable reinfección.

$M Q / A S:$ mefloquina/artesunato; SP/AS: sulfadoxina pirimetamina/artesunato; DHA/PPQ: dihidroartemisinina/piperaquina. 
et al. en el sudeste asiático, donde $11 \%$ de las láminas permanecieron positivas al día $3{ }^{(1)}$. Cabe señalar que en todos los estudios realizados en el Perú la persistencia de parasitemia al día 3 fluctuó entre 0 y 2,6\%.

Al final de la Tabla 1 observamos los resultados de un estudio realizado en la Amazonía, donde los pacientes solo recibieron mefloquina. A diferencia de lo observado con la TC, al día 3 el 25,5\% todavía persistió con parasitemia; por lo tanto, la rapidez en el aclaramiento de la misma se debería a un efecto del artesunato, más que de la mefloquina en la TC.

Este esquema actual estaría amenazado no solo por la posibilidad, en un mundo cada vez más globalizado, de la importación y dispersión de cepas procedentes del sudeste asiático sino también por el riesgo de que una cepa resistente se origine aquí. Las debilidades de nuestro programa de control que harían posible este fenómeno serían las siguientes: 1. La baja cobertura de tratamiento directamente observado (DOT por sus siglas en inglés), ya que diversos estudios lo sitúan entre 40 y $60 \%$ en la Amazonía (7). 2. El esquema usado en el Perú, el cual favorece la monoterapia en caso de abandono. Se administra solo AS el primer día, mientras que el segundo día se incluye mefloquina en una dosis suboptima $(12,5 \mathrm{mg} / \mathrm{kg})$. Según resultados de un estudio de adherencia (Durand comunicación personal) se da tratamiento supervisado al día 2 solo al $70 \%$, y al día 3 al $60 \%$, por lo que en el $30 \%$ de los pacientes que inician tratamiento no se garantiza que hayan continuado con la terapia y estarían recibiendo dosis suboptimas de antimaláricos; por lo tanto, se posibilita la generación de cepas resistentes al artesunato. 3. La reactivación de focos endémicos donde la malaria falciparum ya se había eliminado, como en la costa norte, y por último, 4. La presión de las cepas de países limítrofes; Brasil, Ecuador, Bolivia y Guyana que tienen a la MQ/AS como esquema de primera línea.

Por lo tanto se hace necesario reactivar el sistema de vigilancia de la resistencia a los fármacos antimaláricos en el Perú, mejorar la capacidad del MINSA para administrar tratamiento antimalárico supervisado y modificar el esquema $\mathrm{AS} / \mathrm{MQ}$ al propuesto por la OMS: $\mathrm{AS}+\mathrm{MQ}$ a la dosis de $15 \mathrm{mg} / \mathrm{kg}$ de $\mathrm{MQ}$ el primer día y $10 \mathrm{mg} / \mathrm{kg}$ el segundo día, con la finalidad de administrar un esquema óptimo aun con solo un día de tratamiento, lo cual disminuiría el riesgo de generación de cepas resistentes y, por último, mejorar los planes de contención de brotes de malaria para evitar su dispersión.

En conclusión, tenemos evidencia que hasta el 2006 la TC era eficaz para el tratamiento de la malaria por $P$. falciparum y el artesunato conservaba su propiedad de aclarar rápidamente la parasitemia. La inquietud surge al preguntarnos si esos resultados serían similares si lo aplicamos en la actualidad.

Conflictos de interés: los autores declaran no tener conflictos de interés.

Declaración de responsabilidad: las opiniones y afirmaciones contenidas aquí son propias de los autores y no deben interpretarse como posición oficial o que reflejan la opinión del Ministerio de Salud del Perú, del Departamento de la Marina o del Departamento de Defensa de los Estados Unidos.

\section{REFERENCIAS BIBLIOGRÁFICAS}

1. Rogers WO, Sem R, Tero T, Chim P, Lim P, Muth S, et al. Failure of artesunate-mefloquine combination therapy for uncomplicated Plasmodium falciparum malaria in southern Cambodia. Malar J. 2009;8:10.

2. de Oliveira AM, Chavez J, de Leon GP, Durand S, Arrospide $\mathrm{N}$, Roberts J, et al. Efficacy and effectiveness of mefloquine and artesunate combination therapy for uncomplicated Plasmodium falciparum malaria in the Peruvian Amazon. Am J Trop Med Hyg. 2011;85(3):573-8.

3. Gutman J, Green M, Durand S, Rojas OV, Ganguly B, Quezada WM, et al. Mefloquine pharmacokinetics and mefloquineartesunate effectiveness in Peruvian patients with uncomplicated Plasmodium falciparum malaria. Malar J. 2009;8:58.

4. Grande T, Bernasconi A, Erhart A, Gamboa D, Casapia M, Delgado C, et al. A randomised controlled trial to assess the efficacy of dihydroartemisinin-piperaquine for the treatment of uncomplicated falciparum malaria in Peru. PLoS One. 2007;2(10):e1101.

5. Marquiño W, Ylquimiche L, Hermenegildo Y, Palacios AM, Falconí E, Cabezas C, et al. Efficacy and tolerability of artesunate plus sulfadoxine-pyrimethamine and sulfadoxinepyrimethamine alone for the treatment of uncomplicated Plasmodium falciparum malaria in Peru. Am J Trop Med Hyg. 2005;72(5):568-72.

6. Marquiño W, Huilca M, Calampa C, Falconí E, Cabezas C, Naupay R, et al. Efficacy of mefloquine and a mefloquineartesunate combination therapy for the treatment of uncomplicated Plasmodium falciparum malaria in the Amazon Basin of Peru. Am J Trop Med Hyg. 2003;68(5):608-12.

7. Grietens KP, Soto V, Erhart A, Ribera JM, Toomer E, Tenorio A, et al. Adherence to 7-day primaquine treatment for the radical cure of P. vivax in the Peruvian Amazon. Am J Trop Med Hyg. 2010;82(6):1017-23.

Correspondencia: Salomón Durand

Dirección: Av. La Marina con Trujillo S/N, NAMRU-6 Iquitos

Teléfono: 065-601470

Correo electrónico: salomon.durand@gmail.com 